\title{
Experiencias de aprendizaje en YouTube, un análisis durante la pandemia de COVID-19
}

\author{
Learning experiences on YouTube, \\ an analysis during the COVID-19 pandemic
}

Seira Vera Balderas

Javier Moreno Tapia

\begin{abstract}
RESUMEN
La migración de una educación presencial a una virtual debido a la contingencia sanitaria que se vive en el mundo a causa del coronavirus trajo consigo grandes retos, para enfrentarlos, las tecnologías de la información y comunicación (TIC) representan un elemento clave para los alumnos que utilizan los espacios que han generado para aprender más allá del aula. El objetivo de este artículo es analizar los usos que los estudiantes de una universidad pública en México dan a YouTube durante la pandemia ocurrida en el año 2020 por el COVID-19, e identificar los usos educativos que dan a la herramienta en espacios formales y no-formales de aprendizaje. El enfoque del estudio fue cuantitativo y se utilizó como instrumento para la recolección de los datos un cuestionario elaborado en Google Forms, el cual fue aplicado a 149 estudiantes. Los resultados mostraron que los participantes usan YouTube de manera frecuente para adquirir conocimientos vinculados con diferentes ámbitos de su vida, recuperando aprendizajes valiosos. Se concluye que los estudiantes reconocen a YouTube como un espacio alternativo de aprendizaje, el cual puede ser implementado como recurso en los procesos de enseñanza y aprendizaje en la educación formal.
\end{abstract}

Palabras clave: educación no-formal, TIC, YouTube.

\begin{abstract}
The migration from face-to-face education to a virtual one due to the health contingency that is experienced in the world due to the coronavirus brought great challenges, to face them, information and communication technologies (ICT) represent a key element for students who use the spaces they have generated to learn beyond the classroom. The objective of this article is to analyze the uses that students of a public university in Mexico make of YouTube during the pandemic that occurred in 2020 due to COVID-19, and to identify the educational uses that they give to the tool in formal and nonformal learning spaces. The study approach was quantitative and a questionnaire developed in Google Forms was used as an instrument for data collection, which was applied to 149 students. The results showed that the participants frequently use YouTube to acquire knowledge related to different areas of their life, recovering valuable learning. It is concluded that students recognize YouTube as an alternative learning space, which can be implemented as a resource in the teaching and learning processes in formal education.
\end{abstract}

Keywords: non-formal education, ICT, YouTube.

Recibido: noviembre 15 de 2020 | Aprobado: abril 15 de 2021 | Publicado: mayo 28 de 2021 DOI: https://doi.org/10.33010/ie_rie_rediech.v12i0.1139 


\section{INTRODUCCIÓN}

La forma en que vivimos ha sufrido grandes cambios a raíz de los avances tecnológicos que han surgido; todos los ámbitos de la vida diaria se han reconfigurado por la mediación tecnológica, dando como resultado una nueva sociedad, la denominada sociedad de la información (SI), en la cual las tecnologías de la información y comunicación (TIC) son el elemento básico para su desarrollo (Cabero, 2007).

En la SI las personas tienen la oportunidad de manejar y transmitir grandes cantidades de información de manera casi inmediata gracias al surgimiento de Internet, de la telefonía móvil y de las redes satelitales (López, López y Flores, 2018), cambios que traen consigo retos para las instituciones educativas de México y el mundo que se ven ante la necesidad de adaptarse a esta nueva sociedad.

Como respuesta a ello en el contexto mexicano se ha buscado integrar las TIC al aula, no obstante, su implementación ha sido lenta y presenta muchos obstáculos: las brechas generacionales, la infraestructura de las escuelas, así como el razonamiento utilitario que se les ha impuesto a las TIC en lugar de buscar maneras de integrarlas en todo el proceso de enseñanza y aprendizaje son algunos de ellos (Ruíz y Quintana, 2016; Araujo y Andreu, 2017; López, López y Flores, 2018).

Sumado a todas estas problemáticas, en el 2020 se presentó un reto mayor y sin precedentes: el cierre de escuelas de todos los niveles educativos durante el primer trimestre de ese año debido a la emergencia sanitaria que atraviesa el mundo ocasionada por el COVID-19. ${ }^{1}$ Este hecho significó -y significa- un cambio drástico para docentes y alumnos que dejaron el salón de clases para aprender en un escenario educativo virtual.

Esta transición trae consigo dos grandes desafíos: el primero gira en torno al número, disponibilidad y características de los dispositivos necesarios para realizar las actividades en línea por parte de los docentes y alumnos, y el segundo se relaciona con las habilidades y conocimientos suficientes que deben poseer para poder utilizar

1 Acorde a la ONU (2020), más de 156 millones de estudiantes en América Latina tuvieron que dejar el aula para aprender desde casa.

Seira Vera Balderas. Universidad Autónoma del Estado de Hidalgo, México. Es estudiante de la maestría en Ciencias de la Educación y entre sus publicaciones recientes se encuentra la ponencia "Propuesta de una comunidad virtual de aprendizaje para el aprendizaje del idioma inglés en nivel licenciatura” (2020). Correo electrónico: seira.verab@gmail.com. ID: https:/ /orcid. org/0000-0002-2944-7637.

Javier Moreno Tapia. Profesor-investigador del área académica de Ciencias de la Educación de la Universidad Autónoma del Estado de Hidalgo, México. Es doctor en Tecnología Educativa por la Universidad de Barcelona (España). Tiene reconocimiento al perfil Prodep. Entre sus publicaciones recientes se encuentran los artículos "La autonomía de los estudiantes y las designaciones académicas en el campo universitario" (2020) y "University tutoring, their needs and opportunities in a public institution" (2019). Correo electrónico: javier_moreno@uaeh.edu.mx. ID: https://orcid.org/0000-0003-4029-5440. 
dichos dispositivos de manera efectiva para enseñar y aprender. En este último caso, los profesores han tenido que modificar de un día para otro su forma de trabajo, diseñando e implementando las actividades con una formación digital básica, en la mayoría de los casos, lo que ocasiona que la aplicación no sea la más adecuada (DíazBarriga, 2020).

Paralelamente a los problemas que enfrenta la educación formal debido a la coyuntura actual, los contextos educativos no-formales ${ }^{2}$ e informales ${ }^{3}$ han crecido y ganado protagonismo debido a que responden de mejor manera a los procesos educativos que esta nueva sociedad requiere. Han aprovechado que la información se ha desmaterializado y deslocalizado, al mismo tiempo que se ha liberado a la interacción social de los límites espaciotemporales (Adell, 1997; Cabero, 2007), para crear, procesar y transmitir información de manera sencilla y rápida generando nuevos espacios educativos, o bien utilizando los ya existentes para la educación.

A estos cambios Coll (2013) los denominó como la "nueva ecología del aprendizaje", en la cual, el quién y el dónde se aprende pasaron de un lugar y personas designadas a una multiplicidad de escenarios y agentes; el cuándo dejó de ser exclusivamente al inicio de la vida para instaurarse como un proceso que se lleva durante diferentes etapas de la misma; al qué y para qué se aprende se les agregaron las competencias o habilidades básicas para el siglo XXI y se tomó como fin formar aprendices competentes y capaces de seguir aprendiendo a lo largo de la vida; finalmente, en el cómo se comenzaron a utilizar diferentes lenguajes y formatos de representación de la información, colocando a las TIC como vías de acceso a la información y el conocimiento, lo que lleva a un aprendizaje colaborativo y participativo.

Esto muestra que los llamados "nuevos" procesos, ambientes y agentes educativos no son tan recientes ni surgen a partir de la actual pandemia, ${ }^{4}$ pero se puede observar que, a raíz de ella, se han acelerado aún más estos cambios configurando espacios de aprendizaje alternos que forman parte de la vida diaria de las personas. Un ejemplo de estos es YouTube, plataforma que ha ganado popularidad en el ámbito educativo debido a la creciente disponibilidad de videos destinados a la enseñanza y el aprendizaje que almacena.

2 La educación no-formal es aquella que no pertenece al sistema educativo oficial pero presenta cierto grado de organización, no obstante, tiene mayor flexibilidad de tiempos, espacios, metodologías, modalidades de evaluación y de materiales que la educación formal (Smitter, 2006).

3 La educación informal se desarrolla en las actividades diarias de las personas; el aprendizaje se da a lo largo de toda la vida y mediante este las personas adquieren actitudes, valores, habilidades y conocimientos de la vida cotidiana (Corpas, 2016).

4 Se tienen como antecedentes la enseñanza por correspondencia en el último cuarto del siglo, así como la enseñanza multimedia, telemática y e-learning a mediados de los años noventa (GarcíaAretio, 2013). 
Este sitio web es un servicio gratuito de almacenamiento, administración y difusión de videos en el cual, mediante una cuenta de registro, los usuarios y visitantes pueden subir, buscar y ver el material en cualquier formato de video o audio (Ramírez-Ochoa, 2016). Fue creado en el año 2005 y actualmente cuenta con más de dos mil millones de usuarios por mes en el mundo (YouTube, 2020), además es la tercera red social -como la define la Asociación de Internet- más visitada en México (Asociación de Internet, 2019).

Esta plataforma nació con fines de entretenimiento, sin embargo, a escasos cuatro años de su lanzamiento comenzó a ser utilizada e investigada en diversas disciplinas debido al impacto que tuvo en áreas como la política, el periodismo y la educación, tal como lo demuestra la investigación de Snelson (2011).

En el contexto educativo se ha estudiado la implementación de esta plataforma en los procesos de enseñanza y aprendizaje, demostrando que mejora los resultados académicos de los estudiantes debido a sus características, posibilidades de uso, así como las ventajas y beneficios que ofrece estudiantes y docentes, mismos que se presentan en la tabla 1, la cual se deriva de un análisis minucioso de diversas investigaciones (Ramírez-Ochoa, 2016; Snelson, 2011; Rodríguez y Fernández, 2017; Díaz, Lira, Olguín y Arau, 2018; Barajas, Sifuentes y Ramos, 2018; González, 2018; Abdulrahman, 2016; Watkins y Wilkins, 2011; Ruay y Campos, 2019) y permite observar lo que hace tan atractivo a YouTube para la educación.

Tabla 1. Características, posibilidades educativas de uso y ventajas de YouTube.

\begin{tabular}{|c|c|c|}
\hline Características & Posibilidades educativas de uso & Ventajas/Beneficios \\
\hline Multimediático & Ilustrar conceptos & No requiere suscripción previa \\
\hline Hipertextual & Crear comunidades de aprendizaje & Fácil acceso y uso \\
\hline Pluridireccional & Reforzar los contenidos vistos en clase & Gratuito \\
\hline Independiente & Dinamizar los procesos de enseñanza y aprendizaje & Acceso sin restricción de tiempo o lugar \\
\hline geográfica y & Mejorar el rendimiento académico & Compatibilidad de formatos \\
\hline temporalmente & Fomentar el autoaprendizaje & Motiva a los estudiantes \\
\hline Interactivo & Adquisición de habilidades y destrezas & Es un entorno de aprendizaje más \\
\hline Focalizado & que no se trabajan en el aula & atractivo \\
\hline Actualizado & Indagar información de interés & Hace el aprendizaje más entretenido \\
\hline \multirow[t]{4}{*}{ Social } & Creación de una biblioteca audiovisual & \\
\hline & $\begin{array}{l}\text { Formación de habilidades de búsqueda y } \\
\text { selección de información }\end{array}$ & \\
\hline & Conocer y explorar otros contextos y realidades & \\
\hline & Aprendizaje de idiomas & \\
\hline
\end{tabular}

Fuente: Elaboración propia a partir de los autores mencionados. 
Dentro de esta plataforma los videotutoriales son uno de los formatos preferidos para el aprendizaje, debido a que presenta los contenidos de manera sencilla y directa, en un tiempo reducido, con diversos efectos audiovisuales (González, 2018); además este tipo de recurso es funcional en una sociedad en la que el lenguaje audiovisual está presente en la vida diaria, ya que le permite reforzar los aprendizajes a cualquier persona que viva inmersa en las pantallas digitales. En este sentido, el video tutorial es definido por González (2013) como aquellos "elementos que permiten la parte informativa del proceso de enseñanza de una manera dinámica que atrae al alumno para seguirlo como guía en algún proceso” ( $(10)$.

A pesar de que el video tutorial es relativamente nuevo, el uso del video como recurso educativo no lo es, pero es importante señalar que en el marco de la nueva ecología del aprendizaje y de la sociedad de la información estos formatos están tomando un lugar privilegiado en la educación (Snelson, 2008; Quesada, 2015; Méndez-Giménez, 2018).

Ahora bien, Márquez (1995, citado en Saucedo, Díaz, Herrera y Recio, 2013) plantea que el video tutorial puede favorecer el aprendizaje siempre y cuando:

1. Promueva la adquisición de un aprendizaje significativo mediante el cambio de esquemas de conocimientos previos y la creación de nuevas relaciones entre dichos elementos.

2. Favorezca la adquisición de los conocimientos de una manera interactiva haciendo uso de organizadores gráficos y animaciones.

Por otra parte, el auge de YouTube trajo consigo nuevas figuras: los YouTubers, término con el que se denomina a los creadores de contenido que graban videos sobre diversas temáticas y que administran dichos contenidos en un canal de YouTube (Berzosa, 2017). Entre estos se puede encontrar a los Edutubers, los cuales se centran en producir y compartir videos educativos, llegando a crear comunidades de aprendizaje en torno a su canal.

El impacto, influencia y aportación que están logrando estos personajes en el ámbito educativo ha sido tal que en el 2017 uno de los diez finalistas del Global Teacher Prize fue el español David Calle, creador del canal de YouTube Unicoos, en el cual se encuentran videos de matemáticas, física, química y tecnología, a partir del cual creó una academia en línea, trascendiendo la plataforma.

A partir de lo dicho hasta ahora, se observa que en YouTube se presenta un ecosistema interesante en el que aparecen nuevos formatos y sujetos, no obstante, las investigaciones (Gallego y Murillo, 2018; Kabooha y Elyas, 2018; Pérez, 2014) se han decantado por estudiar la integración de este en el aula antes de analizar la manera en que se llevan a cabo los procesos de enseñanza y aprendizaje en esta plataforma, por ello, la finalidad de este estudio fue analizar los usos que los estudiantes de una universidad pública en México dan a YouTube, puntualmente durante la pandemia 
ocurrida en el año 2020 por el COVID-19, e identificar los usos educativos que dan a la herramienta en espacios formales y no-formales de aprendizaje.

\section{Metodología}

Debido a la naturaleza del objetivo planteado se optó por un estudio descriptivo (Arias, 2012) mediante el cual se buscó caracterizar el perfil de los participantes, así como los dispositivos, tiempos, lugares, contenidos y fines por los que utilizaban YouTube.

La metodología empleada fue de corte cuantitativo y el instrumento utilizado para la recolección de datos fue un cuestionario elaborado en Google Forms, el cual fue piloteado previamente, que constó de 15 preguntas categorizadas en tres apartados: el primero era referente a la edad, género y semestre de los participantes; el segundo se centró en indagar el tiempo, lugares y dispositivos utilizados para ingresar a la plataforma, y el último ahondó en el contenido visto, la frecuencia de consulta de este y su vinculación con los diferentes ámbitos de la vida de los participantes.

Las preguntas fueron en su mayoría cerradas (Alaminos y Castejón, 2016) con escala Likert, sin embargo, para explorar los contenidos y canales específicos, así como su aplicación en la vida diaria de los mismos, se usaron preguntas abiertas.

Para la fiabilidad del cuestionario aplicado se calculó el Alpha de Cronbach a través de la herramienta de análisis de datos cuantitativo SPSS, del cual se obtuvo un valor de .886, que se interpreta como evidencia de que los datos recogidos son confiables.

La población estuvo conformada por una matrícula promedio de 450 estudiantes inscritos oficialmente en la licenciatura en Ciencias de la Educación -según datos del anuario estadístico correspondiente- de una universidad pública del centro de México, a quienes se les envió la invitación para responder el cuestionario vía correo electrónico, de los cuales participaron 149.

El análisis de los datos se realizó mediante estadística descriptiva (Fernández, Cordero y Córdoba, 2002) a través de las aplicaciones Excel y SPSS.

\section{Resultados}

A continuación se describen los hallazgos encontrados a partir de la aplicación del cuestionario.

\section{Perfil de los estudiantes}

Los participantes fueron 124 mujeres y 25 hombres de los diferentes semestres de la licenciatura, con una edad promedio de 20 años.

La edad influyó de manera determinante en los resultados, ya que se trata de nativos digitales quienes, en palabras de Prensky (2001), están acostumbrados a recibir información de manera rápida y con predilección por el lenguaje audiovisual dado 
que nacieron y crecieron rodeados de pantallas; debido a esto, los usos, contenidos y tiempos que pasan en la plataforma son resultado de sus características generacionales.

Por otra parte, el hecho de que la mayoría de los participantes fuera del sexo femenino influyó en los contenidos que visitan en YouTube, marcando una tendencia hacia el contenido de belleza y estilo de vida.

\section{Acceso a YouTube: dispositivos y tiempo de conexión}

El hogar resultó ser el espacio preferido para ingresar a la plataforma, ya que el 69\% mencionó conectarse en este lugar muy frecuentemente, lo cual se relaciona con la red que suelen utilizar, puesto que el 89\% indicó acceder a YouTube mediante la conexión WiFi; estos resultados pueden estar influenciados por dos cosas: en primera instancia, que los estudiantes tienen acceso a internet en el hogar y es por ello que prefieren acceder desde este sitio para no gastar datos móviles, y en segundo lugar, debido a la pandemia los participantes entraron en resguardo domiciliario, lo que los obliga a ver el contenido desde el hogar.

En lo referente a los dispositivos, el celular fue el predilecto al ser utilizado de manera muy frecuente por el 68\% de los estudiantes, seguido por la computadora portátil o laptop, que tuvo un uso frecuente del $43 \%$ de los participantes. Esto refleja que hay una inclinación a los dispositivos móviles debido a que facilitan el acceso en diversos espacios y momentos, tal como lo pronosticaba García-Aretio (2013).

Por otra parte, el 64\% de los estudiantes mencionó ingresar a la plataforma cinco, seis o siete veces a la semana, con un tiempo de conexión promedio de una a dos horas al día, hecho que reafirma los resultados del " $15^{\circ}$ Estudio sobre los hábitos de los usuarios de Internet en México 2018”, donde se menciona que YouTube es la tercera red social preferida de los jóvenes, en la cual pasan gran parte de su tiempo (Asociación de Internet, 2019).

\section{Contenido visto: usos y aprendizajes}

Los resultados apuntan que los estudiantes utilizan la plataforma principalmente para ver contenido de música ( $81 \%$ muy frecuentemente y 15\% frecuentemente), por lo cual predomina su vinculación con el ámbito recreativo, sin embargo, el contenido educativo fue el segundo más visto (33\% muy frecuentemente y 36\% frecuentemente), y aunque los porcentajes no son cercanos, denotan que los participantes son conscientes de las posibilidades educativas que tiene la plataforma y acuden a ella cuando requieren aprender algo.

Además, las respuestas de los estudiantes muestran que, sin importar el tipo de contenido que vean, perciben aprender algo, como se puede observar en la segunda columna de la tabla 2 . Al respecto, únicamente el 2\% de los estudiantes mencionó que solía ver los contenidos pero sin aplicar lo visto, mientras que el 98\% dio algún ejemplo de uso del aprendizaje adquirido. 
Tabla 2. Contenidos y aprendizajes en YouTube.

\begin{tabular}{|c|c|c|c|}
\hline $\begin{array}{l}\text { Contenido } \\
\text { que visitan }\end{array}$ & $\begin{array}{c}\text { Frecuencia de consulta, valoración } \\
\text { del aprendizaje y ámbito de la vida } \\
\text { con el que lo vinculan }\end{array}$ & $\begin{array}{c}\text { Ejemplos } \\
\text { de aprendizaje }\end{array}$ & $\begin{array}{c}\text { Canales } \\
\text { preferidos }\end{array}$ \\
\hline Música & $\begin{array}{l}\text { Lo ven muy frecuentemente. } \\
\text { Consideran aprender mucho a través } \\
\text { de ello y suelen vincularlo con el } \\
\text { ámbito recreativo principalmente }\end{array}$ & $\begin{array}{l}\text { Práctica del idioma inglés mediante } \\
\text { la pronunciación y aprendizaje } \\
\text { de las letras de las canciones }\end{array}$ & $\begin{array}{l}\text { Vevo, AвBA, Camila Cabello, } \\
\text { Pascu y Rodri, One Direction, } \\
\text { Cocomelon, By mila, 5sos }\end{array}$ \\
\hline Viajes & $\begin{array}{l}\text { Lo visualizan ocasionalmente, } \\
\text { aprenden mucho y lo vinculan } \\
\text { con el ámbito recreativo }\end{array}$ & $\begin{array}{l}\text { Conocer sobre la cultura de otros } \\
\text { países, lo que les permite adquirir } \\
\text { conocimientos de cultura general } \\
\text { y entablar conversaciones con } \\
\text { amigos o familiares }\end{array}$ & Luisito Comunica \\
\hline $\begin{array}{l}\text { Belleza y } \\
\text { estilo de vida }\end{array}$ & $\begin{array}{l}\text { Lo ven ocasionalmente, } \\
\text { aprenden poco y lo vinculan } \\
\text { con el ámbito recreativo }\end{array}$ & $\begin{array}{c}\text { Recreación de recetas de cocina } \\
\text { Maquillarse } \\
\text { Seguir rutinas de ejercicio } \\
\text { Compra de productos a partir } \\
\text { de las reseñas } \\
\text { Meditar } \\
\text { Desarrollo de inteligencia emocional }\end{array}$ & $\begin{array}{c}\text { Daniel Habif, De mi rancho a tu } \\
\text { cocina, Toque y sazón, AnnaSarelly, } \\
\text { Gym Virtual, Yuya, Pamela } \\
\text { Robledo, Mariajosegi, Vale HB }\end{array}$ \\
\hline Tecnología & $\begin{array}{l}\text { Lo ven ocasionalmente, } \\
\text { aprenden mucho y lo vinculan } \\
\text { en el ámbito educativo }\end{array}$ & $\begin{array}{l}\text { Uso de aplicaciones y plataformas } \\
\text { Edición de video } \\
\text { Solución de fallas en la computadora }\end{array}$ & No mencionaron ninguno \\
\hline Videojuegos & $\begin{array}{l}\text { Casi nunca lo ven, aprenden } \\
\text { casi nada y no lo vinculan con } \\
\text { algún ámbito de su vida }\end{array}$ & No mencionaron & $\begin{array}{c}\text { Quetzal, Rekhtran, Las reviews de } \\
\text { Camilo, Mauu, El Fedelobo, } \\
\text { Auronplay Cracks Eurogamer }\end{array}$ \\
\hline Salud & $\begin{array}{c}\text { Casi nunca lo ven, aprenden } \\
\text { mucho y lo vinculan con } \\
\text { el ámbito recreativo }\end{array}$ & $\begin{array}{c}\text { Informarse de enfermedades } \\
\text { que padecen }\end{array}$ & Desansiedad \\
\hline Noticias & $\begin{array}{l}\text { Lo ven ocasionalmente, } \\
\text { aprenden mucho y lo vinculan } \\
\text { con el ámbito recreativo }\end{array}$ & $\begin{array}{l}\text { Se informan sobre los sucesos } \\
\text { actuales, lo que les permite conocer } \\
\text { del mundo y discutirlo con sus } \\
\text { amigos y familiares }\end{array}$ & $\begin{array}{c}\text { CNN Noticias, Grupo Fórmula, } \\
\text { El pulso de la república, Noticias } \\
\text { Telemundo, Jacobo Wong, Milenio, } \\
\text { Noticiero en Redes }\end{array}$ \\
\hline $\begin{array}{l}\text { Películas, series, } \\
\text { documentales }\end{array}$ & $\begin{array}{l}\text { Lo ven frecuentemente, } \\
\text { aprenden mucho y lo vinculan } \\
\text { con el ámbito recreativo }\end{array}$ & $\begin{array}{l}\text { Al ver películas o documentales de } \\
\text { temas relacionados con educación, } \\
\text { comprenden mejor algunos temas }\end{array}$ & $\begin{array}{l}\text { History Channel, } \\
\text { National Geographic }\end{array}$ \\
\hline Deportes & $\begin{array}{l}\text { Casi nunca lo ven, aprenden } \\
\text { poco y lo vinculan con } \\
\text { el ámbito recreativo }\end{array}$ & $\begin{array}{c}\text { Informarse sobre sus equipos } \\
\text { o jugadores favoritos }\end{array}$ & Olympic Channel \\
\hline Otros & $\begin{array}{l}\text { Lo ven ocasionalmente, } \\
\text { aprenden poco y lo vinculan } \\
\text { con el ámbito recreativo }\end{array}$ & $\begin{array}{c}\text { Aprender a bailar } \\
\text { Selección de próximas lecturas } \\
\text { por las reseñas vistas } \\
\text { Ideas para generar su } \\
\text { propio contenido } \\
\text { Para vocalizar }\end{array}$ & $\begin{array}{l}\text { Genial, El monitor geek, } \\
\text { WatchMojo, Islas blogs, The top } \\
\text { comics, Los Polinesios, Un poco de } \\
\text { todo, Cordura natural, Pleb snob, } \\
\text { La vida me da acidez, los ADN }\end{array}$ \\
\hline
\end{tabular}


Los resultados apuntan que incluso cuando ven contenido que puede considerarse poco educativo -como la música- y con un propósito de entretenimiento, logran recuperar aprendizajes significativos para ellos. Los conocimientos que los participantes adquieren a través de los videos que consultan en la plataforma son diversos y aplicables para diferentes ámbitos de su vida, como se puede observar en la tercera columna de la tabla 2.

Con referencia a los canales preferidos de consulta se recuperaron 145 diferentes, los cuales se concentran principalmente en el área de "otros" -que corresponde a contenido de entretenimiento a través de videoblogs- y de educación, con $28 \%$ en ambos casos, seguidos por aquellos de belleza y estilo de vida con 16\%. Es importante señalar que en la tabla 2 únicamente se presentan aquellos que se repitieron más de una vez y que resultan representativos del tipo de contenido que se encuentra en la categoría.

En lo que respecta al contenido educativo, se encontró que los estudiantes lo consultan con dos principales objetivos: académico y de interés personal, tal como se muestra en la tabla 3, la cual condensa las respuestas de los participantes en relación con esta categoría.

Tabla 3. Contenido educativo y aprendizajes recuperados.

\begin{tabular}{|c|c|c|}
\hline $\begin{array}{l}\text { Frecuencia de consulta, } \\
\text { valoración de aprendizaje, } \\
\text { ámbito con el que lo } \\
\text { vinculan }\end{array}$ & $\begin{array}{c}\text { Ejemplos } \\
\text { de aprendizaje }\end{array}$ & Canales preferidos \\
\hline \multirow[t]{2}{*}{$\begin{array}{l}\text { Lo ven frecuentemente, } \\
\text { aprenden demasiado } \\
\text { y lo vinculan con el } \\
\text { ámbito académico }\end{array}$} & $\begin{array}{l}\text { Académicos } \\
\text { Realizar tareas } \\
\text { Aclarar dudas } \\
\text { Reforzar temas } \\
\text { Aprender idiomas } \\
\text { Investigar temas académicos } \\
\text { Mejorar su aprendizaje mediante el conocimiento } \\
\text { de técnicas de estudio y estrategias de } \\
\text { aprendizaje } \\
\text { Mejora en su práctica docente a partir del } \\
\text { conocimiento y aplicación de didácticas }\end{array}$ & $\begin{array}{l}\text { Tedx Talks, SuperHolly, Sprouts, Kale Anders, } \\
\text { Un mundo inmenso, Educatina, } \\
\text { YouTube Teachers, Edutopia, Khan academic, } \\
\text { Curiosamente, TV UNAM, Explora tu mundo, } \\
\text { Santos Rivera, La educadora creativa }\end{array}$ \\
\hline & $\begin{array}{l}\text { Interés personal } \\
\text { Adquisición de conocimientos de cultura general } \\
\text { Conocimiento de temas de interés personal que } \\
\text { no se abordan en la escuela }\end{array}$ & $\begin{array}{l}\text { Xpress'Tv, Cocoptero datos, Supercepción, } \\
\text { Histeria, C de ciencia, Doqmentalista, Tri line, } \\
\text { Migala, Píldoras de psicología, Flish Fisher, } \\
\text { KarlaNotes, Tu Cosmopolis, Unibetas, Gran } \\
\text { misterio, Craftingeek, Monitor fantasma, } \\
\text { Esquizofrenia natural, Psicología visual, } \\
\text { Raizarevelles99, Curioso Ben, El pasquín, } \\
\text { El robot de Platón }\end{array}$ \\
\hline
\end{tabular}


Respecto al contenido visto con fines académicos, el 60\% de los estudiantes mencionó que muy frecuentemente busca información o ejemplos que les permitan realizar sus actividades escolares; 37\% lo utiliza frecuentemente para reforzar los temas vistos en clase a través de videos donde se aborden otros puntos de vista o con autores más recientes; $26 \%$ lo ve frecuentemente para realizar tareas y ejercicios buscando bibliografía, resúmenes y explicaciones de las lecturas que deban realizar o seleccionando videos que puedan utilizar para complementar sus exposiciones; 34\% acude a este contenido para aprender a manejar software o plataformas como Word, Excel, SPSS o Schoology.

Es importante señalar que los jóvenes mencionaron ver contenido relacionado con asignaturas como inglés, historia, psicología, matemáticas, filosofía o economía, áreas del conocimiento que suelen complicarse a los alumnos del área de educación debido a ser demasiado teóricas y manejar conceptos con cierto grado de dificultad, hecho que sumado a la repentina mudanza a una educación en línea da como resultado que estos contenidos resulten más complejos de entender, y por ello los estudiantes buscan videos que les expliquen de manera más pausada o interactiva los temas vistos para despejar las dudas que les hayan surgido y que por cuestión de tiempo, flexibilidad del docente o falta de confianza no pudieron externar en clase.

A partir de las respuestas se devela que la vinculación con el ámbito académico se da desde dos roles: a) como estudiantes con relación a las clases que toman, y b) como docentes en formación, ya que comentaron acudir a esta plataforma para conocer dinámicas o contenido específico del nivel educativo en el que realizan sus prácticas profesionales; además, con la pandemia y el cambio de modalidad educativa se vieron en la necesidad de consultar YouTube para aprender a diseñar actividades en ambientes digitales y a usar plataformas como Schoology.

Las respuestas que se presentan en la tabla 3 reflejan claramente los dos roles mencionados. Canales como Super Holly y Kale Anders están vinculados con su papel de estudiantes y específicamente con la materia de inglés, mientras que YouTube Teachers y La educadora creativa se relacionan con su función docente, como recursos de material e ideas para diseñar sus clases.

Es importante enmarcar la incidencia en las respuestas de los TeDx Talks, el cual representó uno de los principales contenidos vinculados con el área de educación que, acorde a lo mencionado por los participantes, les permite conocer experiencias de personajes que se han desempeñado en el área y adquirir conocimientos o nuevos puntos de vista a partir de lo expuesto.

Finalmente, los estudiantes también mencionaron acudir a esta plataforma para conocer y aprender de aquellos temas que no se enseñan en otros espacios, algunas de las respuestas mencionan videos y canales sobre la comunidad LGBT, sobre agricultura urbana o poesía. De esta manera se resume que YouTube ofrece información 
de todo tipo y es una plataforma de servicio que está al alcance de todos, la cual es vista frecuentemente como una fuente de consulta.

\section{Discusión}

A la luz de la revisión de los diversos estudios que se reportan sobre el uso de YouTube, uno de los principales aportes que se distingue es valorar el aprendizaje o uso intencional educativo que aporta la consulta y revisión de los videos en este sitio web.

En este sentido, los estudiantes ven en esta plataforma un entorno disponible donde se puede acceder a todo tipo de información, por lo que, aunque no se trate de un espacio formalmente validado, los estudiantes recurren a él. Esto lo convierte en un área de oportunidad para buscar asesorías o apoyos adicionales a los que por cualquier razón no tiene acceso en el espacio formal de aprendizaje. En términos vygostkianos, se puede inferir que los videos se convierten en un instrumento psicológico que los estudiantes utilizan para aprender, y a través de su uso se ve reflejado el suministro de ayuda pedagógica que bajo otras circunstancias tendría que ofrecer el docente, pero en este caso la labor la cumple un tercero que diseñó y publicó un video que explica el tema, modela procedimientos o incluso es directamente el autor primario de una teoría, ley, modelo o fuente de conocimiento, objeto de estudio dentro de la clase. Es decir, el alumno que por alguna razón no alcanzó a comprender la temática revisada y su zona de desarrollo real se encuentre muy alejada de la zona de desarrollo próximo, gracias al video puede regularizarse y autodirigir su acción para construir el conocimiento esperado (Vygostki, 1979; Coll y Martí, 2002; Coll 2014).

Por lo tanto, YouTube funciona como un espacio para entrar en contacto con asesores de manera asincrónica, los cuales tributan a los aprendizajes promovidos en la educación formal mediante la aclaración de dudas o exposición de ejemplos; de esta manera, YouTube como un contexto de educación no-formal, establece una relación de suplencia con el contexto formal al asumir tareas de este (Smitter, 2006).

Además, la posibilidad que tienen los alumnos de pausar, regresar y repetir el video las veces que lo deseen hasta alcanzar los aprendizajes esperados los hace promover y fortalecer sus competencias de autorregulación y autonomía del aprendizaje, ya que dotan al alumno de insumos de información a los que puede acceder y manipular para la comprensión de un tema de interés, ya sea como un complemento de los contenidos revisado en clase o de cualquier otro contenido de interés (Monereo y Pozo, 2003).

A pesar de estos beneficios, se presenta un gran desafío en relación con la fiabilidad de la información que se consulta, puesto que únicamente dos de los participantes mencionaron utilizar como criterios de selección que fueran de fuentes confiables, por ejemplo, de canales de universidades, o bien que en la descripción del video se encontraran las referencias bibliográficas que dieran validez a lo que el video presenta, lo cual es determinante si se utiliza este contenido con fines académicos. 
Ramírez-Ochoa (2016) planteó que a través del uso de esta plataforma los estudiantes desarrollan sus habilidades de búsqueda y selección de información, no obstante, los hallazgos en este estudio disienten de ello, mostrando que los criterios utilizados se dirigen a aspectos audiovisuales, de temática o número de suscriptores, es decir, de forma más que de contenido.

Es importante conservar estos criterios audiovisuales al utilizar YouTube con fines y en ámbitos educativos porque son los que logran atraer a los estudiantes haciendo el aprendizaje más entretenido y divertido, no obstante, resulta relevante no perder de vista la dimensión pedagógica de los mismos; por ello, es fundamental desarrollar en los alumnos un pensamiento crítico y reflexivo que les permita discernir entre fuentes confiables de consulta de aquellas que no lo son en la vasta colección de videos que se almacenan en esta plataforma, es decir, se deben establecer criterios de validación de los videos de YouTube y de cualquier repositorio audiovisual si se pretende utilizar con fines académicos.

De igual manera, será necesario promover iniciativas para asegurar la rigurosidad en la publicación de videos. Hablamos puntualmente de que todo video que se preste a divulgar información científica debe de poseer cierto número de credenciales, como la autoría, fecha de publicación, citas y referencias consultadas. Ante ello, microespacios como YouTube Edu, en el cual se encuentran videos educativos validados previamente por parte de profesionales, se vuelven muy importantes si se busca informarse con objetivos académicos.

\section{Conclusiones}

YouTube es un espacio virtual que debido a sus características - gratuito, de fácil acceso y uso, multimediático- y a la amplia cantidad de contenidos que almacena, ofrece grandes posibilidades de uso educativo en contextos formales, no-formales e informales, adaptándose al funcionamiento de la sociedad actual, en la cual el aprendizaje se lleva a cabo en diversos espacios, momentos, formatos y con diversos sujetos.

Los estudiantes reconocen a YouTube como un espacio alternativo para el aprendizaje y recurren a él de manera frecuente, debido a esto, ante la actual coyuntura y los desafíos que esta trajo consigo, este sitio web podría implementarse como recurso en los procesos de enseñanza y aprendizaje en la educación formal sin gran dificultad y de manera orgánica, puesto que los alumnos ya están habituados a su uso.

Además, el papel del docente como facilitador del contenido subsanaría la problemática de la fiabilidad de la información, ya que él se encargaría de seleccionar y validar los videos, asegurándose de que estos provengan de fuentes confiables.

Finalmente, a raíz de todo lo expuesto se sugiere que los videos académicos que se realicen utilicen formatos más creativos, agregando animaciones u organizadores gráficos, donde las explicaciones sean breves pero claras, incluyendo ejemplos que 
permitan a los alumnos relacionar los conceptos o temas con la realidad, anexando bibliografía, enlaces o hipertextos que los guíen a las fuentes que avalan la información presentada. De igual manera, se incentiva a los profesores e instituciones educativas a publicar los videos en la plataforma, aumentando así el acervo de videos académicos en YouTube, lo cual sumaría no solo al aprendizaje de sus alumnos sino al de los más de 2 mil millones de usuarios que posee alrededor del mundo, fomentando la creación de comunidades de aprendizaje.

\section{REFERENCIAS}

Abdulrahman Almurashi, W. (2016). The effective use of YouTube videos for teaching english language in classrooms as supplementary material at Taibah University in Alula. International Journal of English Language and Linguistics Research, 4 (3), 32-47. Recuperado de: http:/ /www.eajournals.org/wp-content/uploads/ The-Effective-Use-of-YouTube-Videos-for-TeachingEnglish-Language-in-Classrooms-as-SupplementaryMaterial-at-Taibah-University-in-Alula.pdf.

Adell, J. (1997). Tendencias en educación en la sociedad de las tecnologías de la información. Edutec-e Revista Electrónica de Tecnología Educativa, (7). Recuperado de: https://www.edutec.es/revista/index.php/edutec-e/ article/view/570/299.

Alaminos Chica, A., y Castejón Costa, J. L. (2016). Elaboración, análisis e interpretación de encuestas, cuestionarios y escalas de opinión. Alicante: Marfil/Universidad de Alicante.

Araujo Farias, A., y Andreu Abela, J. (2017). La relación ontológica comunicación/educación en la sociedad del conocimiento y de la información y nuevos desafíos para la docencia. Razón y Palabra, 21 (3), 4-21. Recuperado de: http://www.revistarazonypalabra. org/index.php/ryp/article/view/1039.

Arias Odón, F. G. (2012). El proyecto de investigación. Introducción a la metodología científica (6a. ed.). Caracas: Episteme.

Asociación de Internet (2019). $15^{\circ}$ Estudio sobre los hábitos de los usuarios de Internet en México 2018.

Barajas Montes, E., Sifuentes Godoy, D. A., y Ramos Betancourt, I. (2018). Aprenseñar por medio de YouTube. Una estrategia de enseñanza aprendizaje actual. En J. García, A. Jaik y M. Mejía (coords.), Propuestas didácticas y reflexiones en torno a la mejora de procesos educativos (pp. 32-44). México: Red Durango de Investigadores Educativos A.C.

Berzosa Peñaranda, M. I. (2017). YouTubers y otras especies. El fenómeno que ba cambiado la manera de entender los contenidos audiovisuales. Madrid: Fundación Telefónica/Ariel. Cabero Almenara, J. (2007). Nuevas tecnologías aplicadas a la educación. Madrid: McGraw-Hill.

Coll, C., y Martí, E. (2002). Constructivismo y educación: la concepción constructivista de la enseñanza y el aprendizaje. En C. Coll, J. Palacios y A. Marchesi (coords.), Desarrollo psicológico y educación (t. II, pp. 623652). Madrid: Alianza Editorial.

Coll, C. (2013). El currículo escolar en el marco de la nueva ecología del aprendizaje. Aula de Innovación Educativa, (219), 31-36. Recuperado de: http:/ / diposit. ub.edu/dspace/bitstream/2445/53975/1/627963. pdf.

Coll, C. (2014). El sentido del aprendizaje hoy: un reto para la innovación educativa. Aula de Innovación Educativa, (232), 12-17. Recuperado de: http://diposit. ub.edu/dspace/bitstream/2445/65763/1/642900. pdf.

Corpas, M. D. (2016). La educación informal en el aprendizaje del inglés como lengua extranjera. Tejuelo, (24), 68-91. Recuperado de: https://dialnet.unirioja.es/ servlet/articulo?codigo $=5642224$.

Díaz Azamar, A., Lira Vázquez, I., Olguín Jácome, Z., y Arau Fabián, A. (2018). Enseñanza-aprendizaje a nivel posgrado con la aplicación YouTube. En T. Makita, V. Gaber, J. León y F. Caballero (coords.), Innovación educativa. Avances de cuerpos académicos en casos y aplicaciones (pp. 117-122). México: Red Iberoamericana de Académicas de Investigación A.C. 
Díaz-Barriga, A. (2020). La escuela ausente, la necesidad de replantear su significado. En H. Casanova (coord.), Educación y pandemia. Una visión académica (pp. 19-29). México: UNAM. Recuperado de: http://www.iisue. unam.mx/nosotros/covid/educacion-y-pandemia.

Fernández Fernández, S., Cordero Sánchez, J. M., y Córdoba Largo, A. (2002). Estadística descriptiva. Madrid: Esic.

Gallego Domínguez, C., y Murillo Estepa, P. (2018). La práctica docente mediada con tecnologías. YouTube como herramienta de aprendizaje en educación superior. Foro Educacional, (31), 11-29. Recuperado de: http://ediciones.ucsh.cl/ojs/index.php/ForoEducacional/article/view/1827/1686.

García-Aretio, L. (2013, 16 jul.). De dónde venimos y hacia dónde vamos en educación a distancia. Recuperado de: https:/ / www.YouTube.com/watch?v=J_A2wp7_5Z8\&t=1s.

Global Teacher Prize (2017). David Calle. Recuperado de: https://www.globalteacherprize.org/es / person?id=1703.

González Castelán, Y. (2013). El video tutorial como apoyo pedagógico. Boletín Cientifico Vida Cientifica, 1 (2). Recuperado de: https://www.uaeh.edu.mx/scige/ boletin/prepa4/n1/e8.html.

González, O. (2018). El video tutorial como herramienta de educación no formal en estudiantes de Bogotá, Colombia. Question, 1(59), 1-20. DOI https://doi. org/10.24215/16696581e071.

Kabooha, R., y Elyas, T. (2018). The effects of You'Tube in multimedia instruction for vocabulary learning: Perceptions of EFL students and teachers. English Language Teaching, 11(2), 72-81. Recuperado de: https://eric.ed.gov/?id=EJ1166511.

López de la Madrid, M. C., López de la Madrid, C. A., y Flores Guerrero, K. (2018). Información, conocimiento y aprendizaje en la era digital. Revista de Educación, (15), 119-138. Recuperado de: http:// fh.mdp.edu.ar/revistas/index.php/r_educ/article/ view $/ 2978 / 3240$.

Martín, R. B. (2014). Contextos de aprendizaje: formales, no formales e informales. Ikastorratra, e-Revista de Didáctica, (12), 1-13. Recuperado de: https:/ / dialnet. unirioja.es $/$ servlet/articulo?codigo $=4786184$.

Méndez-Giménez, A. (2018). El enfoque basado en autoconstrucción de materiales. El vídeo-tutorial como estrategia de enseñanza para futuros docentes. Retos: nuevas tendencias en educación física, deporte y recreación, (34), 311-316. Recuperado de: https:/ / dialnet.unirioja.es/ servlet/articulo?codigo $=6736309$.

Monereo, C., y Pozo, J. I. (eds.) (2003). La universidad ante la nueva cultura educativa. Enseñar y aprender para la autonomía. Madrid: Síntesis.

ONU (2020, 26 mar.). Más de 156 millones de estudiantes están fuera de la escuela en América Latina debido a coronavirus: Noticias ONU. Recuperado de: https://news.un.org/ es/story/2020/03/1471822.

Pérez Rubio, J. A. (2014). Enseñanza 2.0: uso de las redes sociales en las prácticas docentes [Tesis de doctorado no publicada]. Universidad Pedagógica Nacional: Colombia.

Quesada Chávez, M. J. (2015). Creación de videos educativos como estrategia didáctica para la formación de futuros docentes de inglés. Actualidades Investigativas en Educación, 15(1), 1-19. http:/ / dx.doi.org/10.15517. v15i1.17588.

Ramírez López, M., y Rodríguez, S. A. (2020). Trayectorias escolares en la educación superior ante la pandemia, ¿continuar, interrumpir o desistir? En H. Casanova (coord.), Educación y pandemia. Una visión académica (pp. 103-108). México: UNAM. Recuperado de: http://www.iisue.unam.mx/nosotros/covid/ educacion-y-pandemia.

Ramírez-Ochoa, M. I. (2016). Posibilidades del uso educativo de YouTube. Ra Ximhai, 12(6), 537 546. Recuperado de: https://www.redalyc.org/ pdf/461/46148194036.pdf.

Rodríguez Villalobos, M. C., y Fernández Garza, J. (2017). Uso del recurso de contenido en el aprendizaje en línea: YouTube. Apertura, 9(1), 22-31. DOI: http:/ / dx.doi.org/10.32870/Ap.v9n1.1018.

Ruay, R., y Campos, E. (2019). La plataforma You'Tube como estrategia para el autoaprendizaje de la lengua inglesa. Boletin REDIPE, 8(12), 129-142. Recuperado de: https:// revista.redipe.org/index.php/1/article/ view/879/805.

Ruíz Torres, V., y Quintana Pareja, E. (2016). De las redes de aprendizaje al aprendizaje en red. Una perspectiva conectiva de la enseñanza de lenguas. Ponencia presentada en Encuentro de Profesores de Español en Escandinavia 2015 (pp. 78-99). Madrid: Instituto Cervantes, Centro Virtual Cervantes. Recuperado de: https://cvc. 
cervantes.es/Ensenanza/biblioteca_ele/publicaciones_centros/PDF/estocolmo_2015/10_ruizquintana.pdf.

Saucedo Fernández, M., Díaz Perera, J. J., Herrera Sánchez, S. C., y Recio Urdaneta, C. E. (2013). El video tutorial como alternativa didáctica en el área de matemáticas. Acta Latinoamericana de Matemática Educativa, 1991-1999. Recuperado de: https://core.ac.uk/download/pdf/20482931.pdf.

Smitter, Y. (2006). Hacia una perspectiva sistémica de la educación no formal. Laurus, 12(22), 241-256. Recuperado de: http://www.redalyc.org/articulo. oa?id $=76102213$.

Snelson, C. (2008). Web-based video education: Possibilities and pitfalls. En Proceedings of TCC 2008, (pp. 214-221). Recuperado de: https://www.learntechlib. org $/ \mathrm{p} / 43828 /$.

Snelson, C. (2011). YouTube across the disciplines: A review of the literature. Merlot Journal of Online Learning and Teaching, 7(1), 159-169. Recuperado de: https://jolt.merlot. org/vol7no1/snelson_0311.pdf.

Vygotski, L. S. (1979). El desarrollo de los procesos psicológicos superiores. Barcelona: Crítica.

Watkins, J., y Wilkins, M. (2011). Using YouTube in the EFL classroom. Language Educaction in Asia, 2(1), 113-119. Recuperado de: http://www.leia.org/LEiA/LEiA\%20 VOLUMES/Download/LEiA_V2_I1_2011/LEiA_ V2I1A09_Watkins_Wilkins.pdf.

YouTube (2020, 14 jul.). YouTube para la prensa. Recuperado de: https://www.youtube.com/intl/es-419/about/press/.

Cómo citar este artículo:

Vera Balderas S., y Moreno Tapia, J. (2021). Experiencias de aprendizaje en YouTube, un análisis durante la pandemia de COVID-19. IE Revista de Investigación Educativa de la REDIECH, 12, e1139. doi: 10.33010/ie_rie_rediech.v12i0.1139. 